SFB/CPP-06-09

TTP06-09

\title{
Complete Higgs mass dependence of top quark pair threshold production to order $\alpha \alpha_{s}$
}

\author{
D. Eiras and M. Steinhauser \\ Institut für Theoretische Teilchenphysik, Universität Karlsruhe \\ 76128 Karlsruhe, Germany
}

\begin{abstract}
In this paper we consider the production of top quark pairs close to threshold and compute the dependence on the Higgs boson mass to order $\alpha \alpha_{s}$. This requires the evaluation of the matching coefficient of the vector current to two-loop level and the inclusion of Higgs mass dependent operators in the non-relativistic effective theory. For Higgs boson masses below $200 \mathrm{GeV}$ moderate contributions to the top quark mass and the peak cross section are observed. We also provide additional information to the on-shell wave function renormalization which is relevant for the matching coefficient.
\end{abstract}

PACS numbers: 14.65.Ha, 14.80.Bn 


\section{Introduction}

One of the most important tasks of a future international linear collider (ILC) [1] is the precise measurement of the cross section for the production of top quark pairs close to threshold. The comparison with theoretical calculations will lead to a determination of the top quark mass with an unrivaled precision leading to an uncertainty below $100 \mathrm{MeV}$ [2]. Furthermore, also the width of the top quark, the strong coupling and the Yukawa coupling between the top quark and Higgs boson can be extracted from such a measurement. The findings of this paper are important in this context. However, the final success of such an enterprise depends crucially on whether the theoretical precision can match the expected experimental one. The complete next-to-next-to-leading analysis of Ref. [3] has shown that it is important to obtain the third-order result within QCD. This constitutes a longterm calculation which has already been started (see, e.g., Refs. [4,5] for the most recent results).

The naive scaling rule $\alpha \sim \alpha_{s}^{2}$, where $\alpha$ is the fine structure and $\alpha_{s}$ is the strong coupling constant, shows that the next-to-next-to-next-to-leading terms are parametrically of the same order as the mixed corrections proportional to $\alpha \alpha_{s}$. In this paper we take the first step to a complete order $\alpha \alpha_{s}$ result and compute the full dependence on the Higgs boson mass. On one hand this requires the evaluation of two-loop vertex corrections within the Standard Model (SM) involving a Higgs boson and a gluon. Furthermore, a new operator depending on the Higgs mass has to be considered on the effective theory side. As we will explicitly see, the two individual pieces are divergent, however, the physical quantities formed by the proper combination is finite.

Effects from the Higgs boson mass have already been studied in Ref. [6] where a Yukawa-type potential has been used and the Schrödinger equation has been solved numerically in order to obtain the imaginary part of the Green function and finally the total cross section. In this paper we will consider the Higgs boson mass to be much larger than the soft scales involved in the process and include it in a systematic way in the non-relativistic effective theory. The importance of a systematic and careful treatment of electroweak corrections to the top quark pair production has been stressed in Ref. [7,8], where certain next-to-next-to-leading logarithmic electroweak effects associated to the instability of the top quark have been considered.

The paper is organized as follows: In the next Section we consider the new Higgs mass dependent operator included into non-relativistic QCD (NRQCD) and evaluate the corrections to the energy level and the wave function at the origin. In Section 3 the Higgs mass dependent corrections to the matching coefficient are discussed and Section 4 contains a brief account of the phenomenological applications of our results. We conclude in Section [5. In Appendix A we discuss the results for the matching coefficients induced by vertex corrections involving a $Z$ boson and Appendix $\mathrm{B}$ contains additional information on the wave function renormalization constant which is relevant in connection to the matching coefficient. 


\section{Effective theory}

The standard theoretical framework for the threshold production of top quark pairs is given by NRQCD $[9,10]$ which ensures the automatic resummation of terms like $\left(\alpha_{s} / v\right)^{n}$ $(n=1,2,3, \ldots)$ where $v$ is the velocity of the produced quarks. NRQCD is constructed from QCD by integrating out the hard scales associated to the mass of the heavy quark, $m$. Thus it contains only degrees of freedom of the order $m v$ and $m v^{2}$.

In this paper we consider next to the top quark, which takes over the role of the heavy quark, also the Higgs boson with mass $M_{h}$. Since $M_{h} \gg m_{t} v$ both mass scales are integrated out simultaneously. In Ref. [11] all operators which appear within QCD up to third order in perturbation theory have been classified. The only operator which has to be added to the Hamiltonian in Eq. (6) of Ref. [11] is given by

$$
\delta \mathcal{H}_{H}=-\frac{\alpha \pi m_{t}^{2}}{s_{W}^{2} M_{W}^{2} M_{H}^{2}},
$$

where $s_{W}$ is the sine of the Weinberg angle and $M_{W}$ is the $W$ boson mass. The corresponding expression in coordinate space is proportional to the delta function. If we employ the counting rule $\alpha \sim \alpha_{s}^{2}$ it is easy to see that $\delta \mathcal{H}_{H}$ gives contributions which are parametrically of the same order as the ones from the third-order QCD Hamiltonian.

Let us in a first step evaluate the corrections to the energy levels induced by the operator $\delta \mathcal{H}_{H}$. Using first order perturbation theory we obtain

$$
\delta E_{n}^{H}=\left\langle\psi_{n}^{C}\left|\delta \mathcal{H}_{H}\right| \psi_{n}^{C}\right\rangle=E_{n}^{C} \frac{\alpha \alpha_{s} C_{F} m_{t}^{4}}{2 s_{W}^{2} n M_{W}^{2} M_{h}^{2}},
$$

with $E_{n}^{C}=-C_{F}^{2} \alpha_{s}^{2} m_{t} /\left(4 n^{2}\right)$ and $\psi_{n}^{C}$ is the Coulomb wave function. $n$ is the principal quantum number.

In order to compute the correction to the wave function at the origin the operator of Eq. (11) has to be inserted into the standard formulae of non-relativistic perturbation theory

$$
\delta \psi_{n}(0)=-\int \mathrm{d}^{3} \vec{r} G_{C}(0, \vec{r}, E) \delta \mathcal{H}_{H} \psi_{n}^{C}(\vec{r}),
$$

where $G_{C}$ is the Coulomb Green function. Following Ref. [12] we split $G_{C}$ into a contribution with zero, one and infinitely many gluon exchanges. Since only the one-gluonexchange part is divergent it is convenient to perform the corresponding calculation in momentum space whereas the other contributions are evaluated in coordinate space. As a final result we obtain

$$
\delta \psi_{n}^{H}(0)=\psi_{n}^{C}(0) \frac{\alpha \alpha_{s} m_{t}^{4}}{s_{W}^{2} M_{W}^{2} M_{h}^{2}} C_{F}\left[-\frac{1}{4} \ln \left(\frac{\alpha_{s} C_{F} m_{t}}{\mu}\right)+\frac{3}{8}\right],
$$

with $\left|\psi_{n}^{C}(0)\right|^{2}=C_{F}^{3} \alpha_{s}^{3} m_{t}^{3} /\left(8 \pi n^{3}\right)$. The divergence in Eq. (41) has been subtracted minimally which will also be done for the coefficient function considered in the next section.

We want to mention that the formulae of this Section are adapted for the top quark. However, they also apply to other quark masses, in particular to the bottom system, by simply exchanging the top quark mass. 


\section{Higgs mass dependence of the matching coefficient}

The matching coefficient of the vector current $j^{\mu}=\bar{t} \gamma^{\mu} t$ is defined by

$$
j_{v}^{k}=c_{v} \phi^{\dagger} \sigma^{i} \chi+\mathcal{O}\left(\frac{1}{m_{t}^{2}}\right),
$$

where $\phi$ and $\chi$ are two-component Pauli spinors for quark and anti-quark, respectively, and $k=1,2,3$ denote the spacial components. Note that there is no contribution to our order from the time-component.

For the practical computation of $c_{v}$ it is convenient to consider the $t \bar{t} \gamma$ vertex in the limit where for the photon energy, $s$, we have $s \approx 4 m_{t}^{2}$. In this case we can establish the equation

$$
Z_{2} \Gamma_{v}=c_{v} \tilde{Z}_{2} \tilde{Z}_{v}^{-1} \tilde{\Gamma}_{v}+\ldots
$$

where we have on the left- and right-hand side quantities of the full and effective theory, respectively. The latter are marked by a tilde and the ellipses denote terms suppressed by inverse powers of the top quark mass. $\Gamma_{v}$ denotes the $t \bar{t} \gamma$ vertex corrections where it is understood that the couplings and masses are renormalized. The two-loop mixed correction to the on-shell wave function renormalization, $Z_{2}$, has been computed recently in Ref. [13]. In particular, for the Higgs boson contributions both the exact result and the expansions in three kinematic regions have been provided.

At this point it is convenient to apply the so-called threshold expansion $[14,15]$ to Eq. (6) which has the consequence that $\Gamma_{v}$ has to be evaluated for $s=4 m_{t}^{2}$ since all except the hard region cancel in Eq. (6). Furthermore, on the right-hand side only tree contributions have to be considered. In particular we have $\tilde{Z}_{2}=1$.

The one-loop corrections to $c_{v}$ are finite [16]. However, starting from two-loop order, the matching coefficient exhibits infra-red divergences which are compensated by ultraviolet divergences of the effective theory rendering physical quantities finite. In Eq. (6) the renormalization constant $\tilde{Z}_{v}$ which generates the anomalous dimension of $\tilde{j}_{v}$ takes over this part. Note that the vector current in the full theory does not get renormalized.

It is convenient to introduce the perturbative decomposition of the matching coefficient

$$
c_{v}=1+\frac{\alpha_{s}}{\pi} C_{F} c_{v}^{\mathrm{QCD}, 1}+\left(\frac{\alpha_{s}}{\pi}\right)^{2} C_{F} c_{v}^{\mathrm{QCD}, 2}+\frac{\alpha}{\pi s_{W}^{2}} c_{v}^{\mathrm{ew}}+\frac{\alpha \alpha_{s}}{\pi^{2} s_{W}^{2}} C_{F} c_{v}^{\mathrm{mix}},
$$

where analog formulae also hold for $Z_{2}$ and $\Gamma_{v}$. Since we consider in this paper only the contribution from the Higgs boson the corresponding quantities obtain an additional superscript $H$.

The two-loop QCD corrections, $c_{v}^{\mathrm{QCD}, 2}$, have been computed in Refs. [17,18] (see also Ref. [19]). The complete SM corrections to one-loop order can be found in Ref. [16] where next to the vertex corrections also the box diagrams contributing to $e^{+} e^{-} \rightarrow t \bar{t}$ have been considered. The latter, however, get no contributions from the Higgs boson.

From the perturbative expansion of Eq. (6) it is easy to see that the one-loop results $c_{v}^{\mathrm{QCD}, 1}$ and $c_{v}^{\mathrm{ew}}$ are simply given by the sum of the one-loop expressions for $Z_{2}$ and $\Gamma_{v}$. 
Whereas the individual pieces are still divergent the proper sum is finite. For convenience we repeat the QCD corrections and the Higgs mass dependent term of $c_{v}^{\mathrm{ew}}$ which are given by

$$
\begin{aligned}
c_{v}^{\mathrm{QCD}, 1} & =-2 \\
c_{v}^{H, \mathrm{ew}} & =\frac{m_{t}^{2}}{M_{W}^{2}}\left[\frac{3 y_{H}^{2}-1}{12 y_{H}^{2}}-\frac{2-9 y_{H}^{2}+12 y_{H}^{4}}{48 y_{H}^{4}} \ln y_{H}^{2}-\frac{\left(-2+5 y_{H}^{2}-6 y_{H}^{4}\right)}{24 y_{H}^{2}} \Psi\left(y_{H}\right)\right],
\end{aligned}
$$

where

$$
\Psi(x)= \begin{cases}\frac{\sqrt{4 x^{2}-1}}{x^{2}} \arctan \sqrt{4 x^{2}-1} & \text { for } x \geq \frac{1}{2} \\ \frac{\sqrt{1-4 x^{2}}}{2 x^{2}} \ln \frac{1-\sqrt{1-4 x^{2}}}{1+\sqrt{1-4 x^{2}}} & \text { for } x<\frac{1}{2}\end{cases}
$$

and $y_{H}=m_{t} / M_{h}$.

At two-loop order it is quite difficult to obtain a closed analytic result valid for all Higgs and top quark masses. However, it is possible to get compact formulae valid in various kinematical regions which — when combined — cover the whole Higgs mass range. This strategy has been successfully applied in Ref. [13] to the on-shell wave function

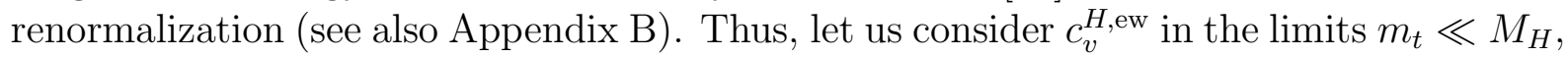
$m_{t} \approx M_{H}$ and $m_{t} \gg M_{H}$ where it is given by

$$
\begin{aligned}
c_{v, 0}^{H, \mathrm{ew}}= & \frac{m_{t}^{2}}{M_{W}^{2}}\left[y_{H}^{2}\left(\frac{31}{144}-\frac{5}{24} \ln y_{H}^{2}\right)+y_{H}^{4}\left(-\frac{3}{16}-\frac{\ln y_{H}^{2}}{4}\right)+y_{H}^{6}\left(-\frac{307}{480}-\frac{5 \ln y_{H}^{2}}{8}\right)\right. \\
& \left.+y_{H}^{8}\left(-\frac{737}{360}-\frac{11 \ln y_{H}^{2}}{6}\right)+\ldots\right], \\
c_{v, 1 a}^{H, \mathrm{ew}}= & \frac{m_{t}^{2}}{M_{W}^{2}}\left[\frac{1}{6}+\frac{\pi}{8 \sqrt{3}}+y_{H, 1 a}\left(\frac{1}{24}+\frac{\pi}{8 \sqrt{3}}\right)+y_{H, 1 a}^{2}\left(-\frac{1}{12}+\frac{\pi}{6 \sqrt{3}}\right)\right. \\
& \left.+y_{H, 1 a}^{3}\left(-\frac{1}{36}+\frac{13 \pi}{108 \sqrt{3}}\right)+y_{H, 1 a}^{4}\left(\frac{1}{288}+\frac{5 \pi}{54 \sqrt{3}}\right)+\ldots\right], \\
c_{v, 1 b}^{H, \mathrm{ew}}= & \frac{m_{t}^{2}}{M_{W}^{2}}\left[\frac{1}{6}+\frac{\pi}{8 \sqrt{3}}-y_{H, 1 b}\left(\frac{1}{24}+\frac{\pi}{8 \sqrt{3}}\right)+y_{H, 1 b}^{2}\left(-\frac{1}{8}+\frac{\pi}{24 \sqrt{3}}\right)\right. \\
& \left.-y_{H, 1 b}^{3}\left(\frac{13}{72}-\frac{19 \pi}{216 \sqrt{3}}\right)+y_{H, 1 b}^{4}\left(-\frac{59}{288}+\frac{23 \pi}{216 \sqrt{3}}\right)+\ldots\right], \\
c_{v, \infty}^{H, \mathrm{ew}}= & \frac{m_{t}^{2}}{M_{W}^{2}}\left[\frac{\pi}{4} y_{H}-\frac{\ln y_{H}}{2}-\frac{23 \pi}{96} \frac{1}{y_{H}}+\left(\frac{7}{48}+\frac{3}{8} \ln y_{H}\right) \frac{1}{y_{H}^{2}}+\frac{55 \pi}{512} \frac{1}{y_{H}^{3}}\right. \\
& \left.+\left(-\frac{71}{720}-\frac{\ln y_{H}}{12}\right) \frac{1}{y_{H}^{4}}+\ldots\right],
\end{aligned}
$$




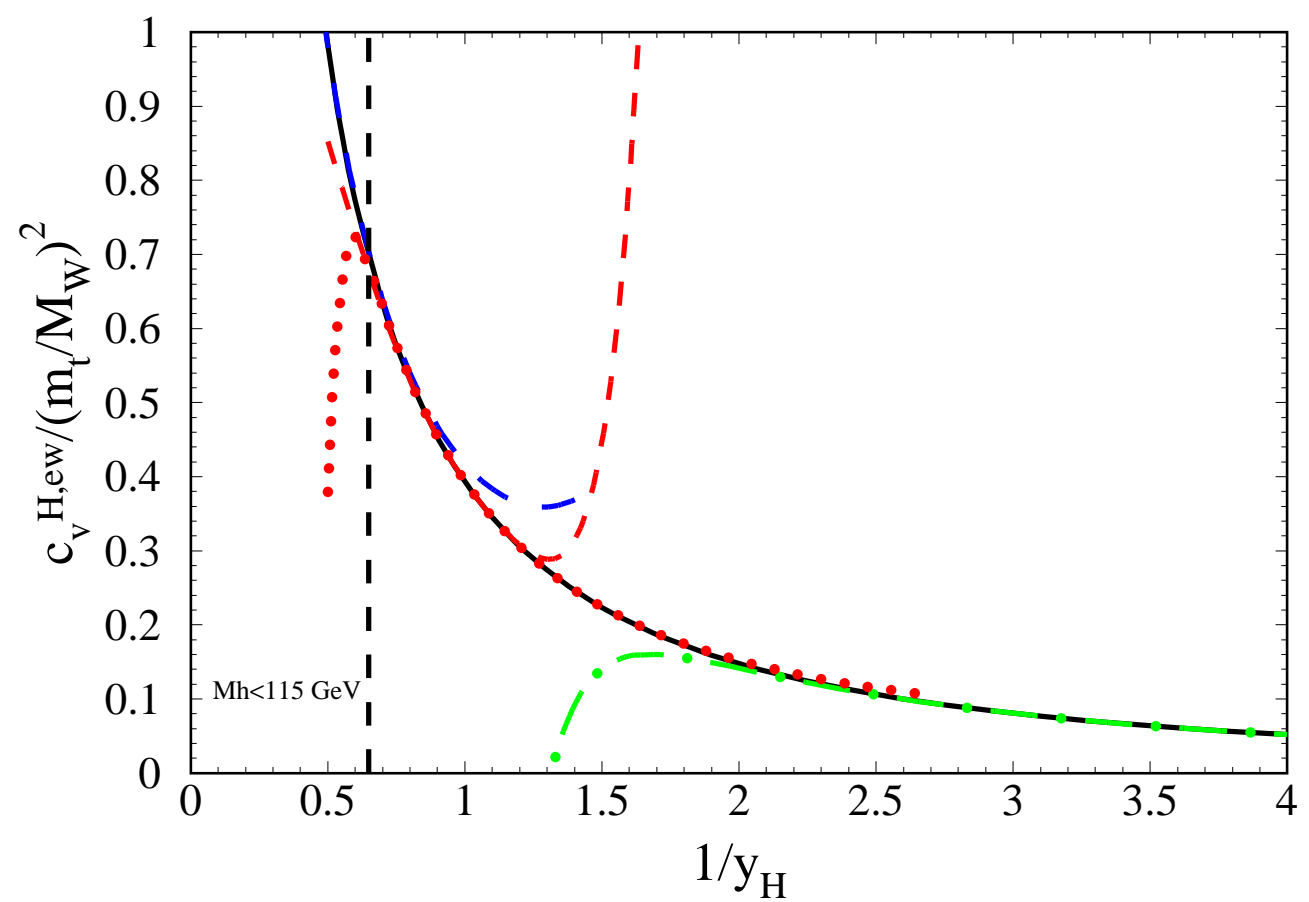

Figure 1: $c_{v}^{H, \mathrm{ew}} /\left(m_{t}^{2} / M_{W}^{2}\right)$ as a function of $1 / y_{H}$. The solid (black) line represents the exact result. The approximations in the three regions are shown as dotted, dashed and dash-dotted lines where for the expansion around $y_{H} \approx 1$ two different approximations have been chosen.

with $y_{H, 1 a}=\left(1-1 / y_{H}^{2}\right)$ and $y_{H, 1 b}=\left(1-y_{H}^{2}\right) . \quad c_{v, 1 a}^{H, \text { ew }}$ and $c_{v, 1 b}^{H, \text { ew }}$ are two different representations of the same information which turn out to be useful for different values of $y_{H}$.

In Fig. [1 the exact result for $c_{v}^{\mathrm{ew}} /\left(m_{t}^{2} / M_{W}^{2}\right)$ (full black line, cf. Eq. (8) ) is shown together with the expansions from Eq. (10), $c_{v, 0}^{H, \text { ew }}$ (dash-dotted), $c_{v, 1 a}^{H, \text { ew }}$ (short dashed), $c_{v, 1 b}^{H, \text { ew }}$ (dotted) and $c_{v, \infty}^{H, \text { ew }}$ (long dashed). One can see that the approximations nicely cover the whole region of $y_{H}$. Note that the expansion around $m_{t}=M_{H}$ shows better convergence properties in case $y_{H, 1 b}$ is used as parameter.

Let us draw the attention of the reader to the expansion terms for large top quark masses which starts with an enhancement factor $m_{t}^{3} /\left(M_{W}^{2} M_{h}\right)$. Two out of the three powers in $m_{t}$ come from the Yukawa coupling between the Higgs boson and the top quark and the factor $m_{t} / M_{h}$ is the indication of the Coulomb singularity which would be present for a massless Higgs boson. The next-to-leading term is quadratic in $m_{t}$ accompanied by a logarithm in $m_{t} / M_{h}$. Both terms indicate that even for the leading terms it is not possible to nullify the Higgs boson mass and non-trivial integration regions have to be considered 

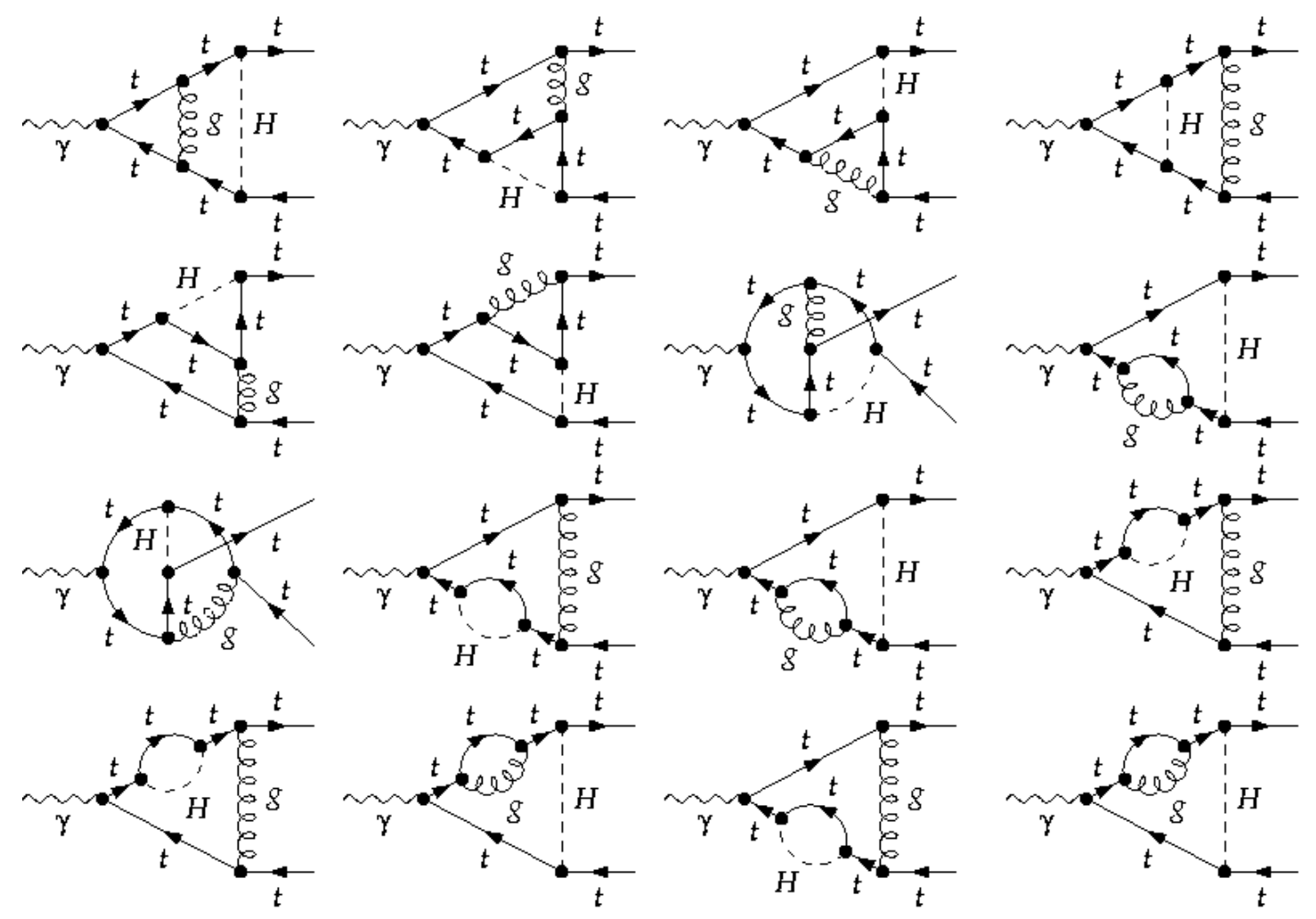

Figure 2: Two-loop Feynman diagrams contributing to $c_{v}^{H \text { mix }}$.

which makes the evaluation of the corresponding expansion terms quite tedious. This is particularly true for the two-loop order where due to the Coulomb divergence one will have a further factor $m_{t} / M_{h}$ as compared to the one-loop term. Thus the expansion starts with a quartic top quark mass dependence. Furthermore, there are momentum regions which have $\sqrt{m_{t} / M_{H}}$ as expansion parameter.

On the other hand, as can be seen in Fig. 11, the result from this region is phenomenologically less important since for Higgs boson masses above $115 \mathrm{GeV}$ there is perfect agreement between the exact result and the approximation one obtains for $m_{t} \approx M_{h}$ and $m_{t} \ll M_{h}$. Higgs boson masses below approximately $115 \mathrm{GeV}$ are excluded by the direct search at the CERN Large Electron Positron Collider (LEP). For this reason we do not consider the limit $m_{t} \gg M_{h}$ at two-loop order.

Let us now turn to two loops. The Feynman diagrams are shown in Fig. 2. They are generated with QGRAF [20]. The application of q2e and exp [21,22] identifies the topology of the individual diagrams and adopts the notation in order to match one of the following 
functions

$$
\begin{aligned}
& J^{ \pm}\left(n_{1}, n_{2}, n_{3}, n_{4}, n_{5}\right)= \\
& \quad \frac{e^{2 \epsilon \gamma_{E}}}{\left(i \pi^{d / 2}\right)^{2}} \int \frac{\mathrm{d}^{d} k \mathrm{~d}^{d} l}{\left(k^{2}+2 k q\right)^{n_{1}}\left(l^{2} \pm 2 l q\right)^{n_{2}}\left(k^{2}\right)^{n_{3}}\left((k-l)^{2}\right)^{n_{4}}\left(l^{2}-M^{2}\right)^{n_{5}}}, \\
& H_{N}^{ \pm}\left(n_{1}, n_{2}, n_{3}, n_{4}, n_{5}\right)= \\
& \quad \frac{e^{2 \epsilon \gamma_{E}}}{\left(i \pi^{d / 2}\right)^{2}} \int \frac{\mathrm{d}^{d} k \mathrm{~d}^{d} l}{\left(k^{2}+2 k q\right)^{n_{1}}\left(l^{2} \pm 2 l q\right)^{n_{2}}\left(k^{2}\right)^{n_{3}}\left((k-l)^{2}-M^{2}\right)^{n_{4}}\left(l^{2}\right)^{n_{5}}}, \\
& Y_{N}^{ \pm}\left(n_{1}, n_{2}, n_{3}, n_{4}, n_{5}\right)= \\
& \quad \frac{e^{2 \epsilon \gamma_{E}}}{\left(i \pi^{d / 2}\right)^{2}} \int \frac{\mathrm{d}^{d} k \mathrm{~d}^{d} l}{\left(k^{2}+2 k q\right)^{n_{1}}\left(l^{2} \pm 2 l q\right)^{n_{2}}\left((k-l)^{2} \mp 2 q(k-l)\right)^{n_{3}}\left(k^{2}\right)^{n_{4}}\left(l^{2}-M^{2}\right)^{n_{5}}}, \\
& Z_{N}^{+}\left(n_{1}, n_{2}, n_{3}, n_{4}, n_{5}\right)= \\
& \quad \frac{e^{2 \epsilon \gamma_{E}}}{\left(i \pi^{d / 2}\right)^{2}} \int \frac{\mathrm{d}^{d} k \mathrm{~d}^{d} l}{\left(k^{2}+2 k q\right)^{n_{1}}\left(l^{2}+2 l q\right)^{n_{2}}\left((k-l)^{2}-2 q(k-l)\right)^{n_{3}}\left(k^{2}-M^{2}\right)^{n_{4}}\left(l^{2}\right)^{n_{5}}}
\end{aligned}
$$

where $d=4-2 \epsilon$ is the space-time dimension and the $n_{i}$ are integer indices. The choice of the five-line integrals of Eq. (11) is possible due to the special kinematics we have at hand. Note that $J^{+}, H^{+}$and $Y^{-}$correspond to self energies whereas $J^{-}, H^{-}, Y^{+}$and $Z^{+}$to vertex diagrams.

In a next step we use the program AIR [23] in order to reduce the integrals to about 30 master integrals. They range from very simple two-point expressions up to complicated two-scale integrals with five lines. At this point an asymptotic expansion in the various kinematical regions is performed. From the one-loop result (cf. Fig. 1) and from the considerations in the context of the on-shell wave function renormalization (see Ref. [13] and Appendix $(\mathrm{B})$ one can see that for the phenomenological interesting Higgs boson masses it is sufficient to have the expansion around $m_{t} \approx M_{H}$ and the one for $m_{t} \ll$ $M_{H}$ at hand. Thus the master integrals are expanded in these two limits. In the case $m_{t} \approx M_{H}$ this reduces to a simple Taylor expansion whereas for $m_{t} \ll M_{H}$ the wellestablished hard-mass procedure [15] is applied. The latter is actually automated in the program exp $[21,22]$. Hence as an independent check we applied with the help of exp the hard-mass procedure to each diagram which immediately leads to simpler expressions and avoids the reduction to the master integrals. In this way the result for $m_{t} \ll M_{h}$ could be checked. We want to mention that the calculation has been performed for general QCD gauge parameter, $\xi$. The cancellation of $\xi$ in the final result serves as a welcome check for our calculation.

As already mentioned in the Introduction, even after the proper combination of $Z_{2}$ and $\Gamma_{v}$ is formed, as prescribed by Eq. (6) , there remains an infra-red divergence which is plugged into $\tilde{Z}_{v}$. We subtract this divergence in the $\overline{\mathrm{MS}}$ scheme and introduce the anomalous dimension $\gamma_{v}=\frac{\mathrm{d} \ln \tilde{Z}_{v}}{\mathrm{~d} \ln \mu}$ which is given by

$$
\gamma_{v}=-\frac{\alpha \alpha_{s}}{\pi^{2} s_{W}^{2}} C_{F} \frac{\pi^{2}}{4} \frac{m_{t}^{4}}{M_{W}^{2} M_{h}^{2}} .
$$


In the regions $m_{t} \ll M_{H}$ and $m_{t} \approx M_{H}$ three, respectively, six expansion terms have been evaluated. Our results read

$$
\begin{aligned}
c_{v, 0}^{H, \text { mix }}= & \frac{m_{t}^{2}}{M_{W}^{2}}\left[\left(\frac{\pi^{2}}{8} \ln \frac{m_{t}^{2}}{\mu^{2}}-\frac{29}{216}-\frac{277 \pi^{2}}{2304}-\frac{\pi^{2} \ln 2}{8}-\frac{21 \zeta_{3}}{16}+\frac{139}{216} \ln y_{H}^{2}\right.\right. \\
& \left.-\frac{103}{288} \ln ^{2} y_{H}^{2}\right) y_{H}^{2}+\left(\frac{583}{576}-\frac{875 \pi^{2}}{6912}+\frac{151}{192} \ln y_{H}^{2}-\frac{17}{16} \ln ^{2} y_{H}^{2}\right) y_{H}^{4} \\
& \left.+\left(\frac{1533691}{432000}-\frac{27103 \pi^{2}}{138240}-\frac{66647}{43200} \ln y_{H}^{2}-\frac{2251}{720} \ln ^{2} y_{H}^{2}\right) y_{H}^{6}+\ldots\right], \\
c_{v, 1 a}^{H, \text { mix }}= & \frac{m_{t}^{2}}{M_{W}^{2}}\left[\frac{\pi^{2}}{8} \frac{1}{1-y_{H, 1 a}} \ln \frac{m_{t}^{2}}{\mu^{2}}-5.760-5.533 y_{H, 1 a}\right. \\
& \left.-5.704 y_{H, 1 a}^{2}-5.888 y_{H, 1 a}^{3}-6.053 y_{H, 1 a}^{4}-6.200 y_{H, 1 a}^{5}+\ldots\right], \\
c_{v, 1 b}^{H, \text { mix }}= & \frac{m_{t}^{2}}{M_{W}^{2}}\left[\frac{\pi^{2}}{8}\left(1-y_{H, 1 b}\right) \ln \frac{m_{t}^{2}}{\mu^{2}}-5.760+5.533 y_{H, 1 b}-0.171 y_{H, 1 b}^{2}\right. \\
& \left.+0.0124 y_{H, 1 b}^{3}+0.0304 y_{H, 1 b}^{4}+0.0296 y_{H, 1 b}^{5}+\ldots\right],
\end{aligned}
$$

where $m_{t}$ is the on-shell mass and $\zeta_{3} \approx 1.20206$ is Riemann's zeta-function. The $\ln \left(m_{t}^{2} / \mu^{2}\right)$ term reminds on the divergence which has been subtracted minimally. Since in the limit $m_{t} \ll M_{H}$ some coefficients of the $\epsilon$-expansion of the master integrals could only be computed numerically the results for the finite parts of $c_{v, 1 a}^{H \text {,mix }}$ and $c_{v, 1 b}^{H \text {,mix }}$ are presented in numerical form. We also want to stress again that $c_{v, 1 a}^{H \text {,mix }}$ and $c_{v, 1 b}^{H \text {,mix }}$ contain the same information. However, expressed in terms of $y_{H, 1 b}$ the convergence properties are much better.

In Fig. 3 the results are shown where the same notation as for the one-loop result has been adopted and $\mu=m_{t}$ has been chosen. Next to the highest expansion terms we also include the lower-order terms as thin lines which nicely demonstrates the convergence properties in the individual regions. Comparing the two parameterizations of the expansion around $M_{t}=M_{h}$ one observes that the one in terms of $y_{H, 1 b}$ demonstrates a much better convergence behaviour: the thin dots in Fig. 3 are practically indistinguishable from the fat ones. This can also be seen in Eq. (13) where the coefficients become quite small starting from the third term which is not the case for $c_{v, 1 a}^{H, \text { mix }}$. Thus, the combination of $c_{v, 1 b}^{H, \text { mix }}$ and $c_{v, 0}^{H, \text { mix }}$ provide a very good approximation to $c_{v}^{H}$ for Higgs boson masses above $M_{h}=115 \mathrm{GeV}$. 


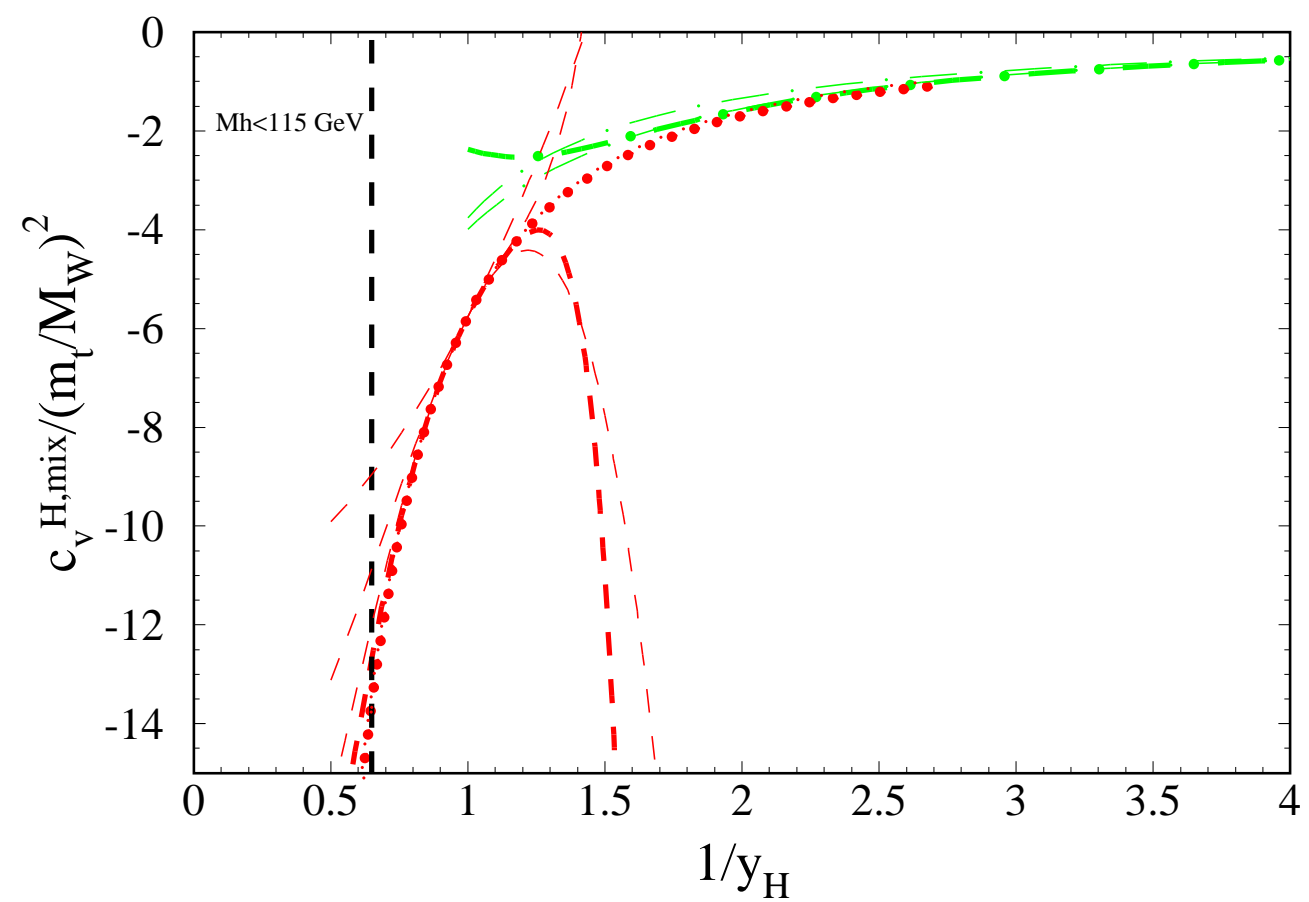

Figure 3: $c_{v}^{\mathrm{mix}}$ as a function of $1 / y_{H}$. The approximations are shown as dotted, dashed and dash-dotted lines where for the expansion around $y_{H} \approx 1$ two different parameterizations have been chosen. Lower-order terms are plotted using thin lines. For the renormalization scale $\mu=m_{t}$ has been adopted.

\section{Phenomenological application}

Let us in a first step discuss the effect on the top quark mass. The connection between the position of the peak in the threshold cross section, $E_{\text {res }}$, and the top quark mass is given by

$$
E_{\mathrm{res}}=2 m_{t}+E_{1}^{\mathrm{p} . \mathrm{t} .}+\delta^{\Gamma_{t}} E_{\mathrm{res}}
$$

where $E_{1}^{\text {p.t. }}$ is the perturbative part of the ground state energy and $\delta^{\Gamma_{t}} E_{\text {res }}=100 \pm$ $10 \mathrm{MeV}[24]$ takes into account the effect of the finite width, the higher order resonances and the continuum. Non-perturbative effects are negligible for the top quark system. $E_{1}^{\text {p.t. }}$ up to third order in QCD has been computed in Ref. [24]. The contribution from the Higgs boson is given in Eq. (2) where $\alpha_{s}$ has to be evaluated at the typical soft scale given by $\mu_{s}=C_{F} \alpha_{s}\left(\mu_{s}\right) m_{t} \approx 30 \mathrm{GeV}$.

According to Eq. (14) a shift in $E_{1}^{\text {p.t. }}$ can directly be translated into a shift in the top quark mass, $\delta m_{t}$. Choosing for the input values $m_{t}=175 \mathrm{GeV}, M_{W}=80.41 \mathrm{GeV}$, $s_{W}^{2}=0.23, \alpha=1 / 127[25], \alpha_{s}\left(M_{Z}\right)=0.118, M_{H}=120 \mathrm{GeV}$ and $\mu_{s}=30 \mathrm{GeV}$ we observe 
for $\delta m_{t} \approx-E_{1}^{H} / 2 \approx 26 \mathrm{MeV}$. This reduces to $9(1) \mathrm{MeV}$ for $M_{H}=200(500) \mathrm{GeV}$. If $M_{H}=120 \mathrm{GeV}$ is adopted and $\mu_{s}=15(60) \mathrm{GeV}$ is chosen one obtain $\delta m_{t} \approx 38(18) \mathrm{MeV}$. Thus, for light Higgs masses relatively large effects are observed whereas for larger $M_{H}$ the numerical effect on $m_{t}$ is small. Note that our findings are in agreement with the more qualitative analysis of Ref. [6].

As a second application it is interesting to consider the effect of the new corrections to the normalized cross section $R=\sigma\left(e^{+} e^{-} \rightarrow t \bar{t}\right) / \sigma\left(e^{+} e^{-} \rightarrow \mu^{+} \mu^{-}\right)$at the resonance energy which is dominated by the contribution from the would-be toponium ground-state. It is of the form

$$
R_{1}=R_{1}^{\mathrm{LO}} c_{v}^{2}\left(m_{t}\right) \frac{\left|\psi_{1}(0)\right|^{2}}{\left|\psi_{1}^{C}(0)\right|^{2}}+\ldots
$$

with $R_{1}^{\mathrm{LO}}=6 \pi N_{c} Q_{t}^{2}\left|\psi_{1}^{C}(0)\right|^{2} /\left(m_{t}^{2} \Gamma_{t}\right)$. The ellipses in Eq. (15) denote contributions from higher order operators. Note that the divergences in $c_{v}$ and $\psi_{1}(0)$ cancel in the combination of Eq. (15). The most advanced evaluation of $R_{1}$ is provided in Ref. [4] where all logarithmically enhanced third-order corrections and the ones proportional to $\beta_{0}^{3}$ are included. Let us for completeness repeat the final numerical result which is given by

$$
R_{1} \approx R_{1}^{\mathrm{LO}}\left(1-0.244_{\mathrm{NLO}}+0.449_{\mathrm{NNLO}}-0.277_{\mathrm{N}^{3} \mathrm{LO}^{\prime}}+\delta_{H}^{(1)}+\delta_{H}^{(2)} \ldots\right),
$$

where $\mu_{s}=30 \mathrm{GeV}$ has been chosen. The prime reminds that the $\mathrm{N}^{3} \mathrm{LO}$ corrections are not complete and $\delta_{H}^{(1)}$ and $\delta_{H}^{(2)}$ parameterize the one- and two-loop corrections due to the Higgs boson considered in the present paper. Using $M_{H}=120 / 200 / 500 \mathrm{GeV}$ and $\mu_{s}=30 \mathrm{GeV}$ we obtain $\delta_{H}^{(1)}=0.067 / 0.034 / 0.009$ and $\delta_{H}^{(2)}=0.036 / 0.011 / 0.0002$. Thus, moderate effects are observed for Higgs boson masses below about $200 \mathrm{GeV}$. However, in contrast to the pure QCD effects of Eq. (16) the convergence seems to be much better as $\delta_{H}^{(2)}$ it is substantially smaller than $\delta_{H}^{(1)}$.

In principle it is possible to apply the formulae of this Section also to the bottom system. However, due to the suppression factor $m_{b}^{2} / M_{H}^{2}$ the numerical effect is very small for Higgs boson masses above $100 \mathrm{GeV}$.

\section{Conclusions}

In this paper we take an important step towards the evaluation of the mixed QCD/electroweak corrections to the threshold production of top quark pairs, namely, the complete Higgs boson mass dependence is computed. In particular, the two-loop corrections to the matching coefficients are evaluated and the corresponding operator is introduced in the effective theory. Moderate numerical effects on the top quark mass and the peak cross section are observed for Higgs boson masses below $200 \mathrm{GeV}$. Considering the anticipated precision of an ILC it is certainly necessary to take into account these corrections.

\section{Acknowledgements}

We would like to thanks J.H. Kühn, A.A. Penin, V.A. Smirnov and O.V. Tarasov for 
useful discussions and A.A. Penin for carefully reading the manuscript. This work was supported by the "Impuls- und Vernetzungsfonds" of the Helmholtz Association, contract number VH-NG-008 and the SFB/TR 9.

\section{A Matching coefficients for the $Z$ boson exchange di- agrams}

In the following we present the results for the matching coefficients induced by vertex corrections involving the $Z$ boson and the corresponding Goldstone boson, respectively. The calculation is similar to the diagrams involving a Higgs boson which is presented in the main part of the paper. Note that there are also box diagrams involving the $Z$ boson which are not considered here. The one-loop result is given by [16]

$$
\begin{aligned}
c_{v}^{Z, \text { ew }}= & a_{t}^{2} s_{W}^{2}\left[\frac{5 y_{Z}^{2}-2}{12 y_{Z}^{2}}-\frac{3 y_{Z}^{4}-4 y_{Z}^{2}+1}{12 y_{Z}^{4}} \ln y_{Z}^{2}+\frac{\left(y_{Z}^{2}-1\right)^{2}}{6 y_{Z}^{2}} \Psi\left(y_{Z}\right)\right] \\
& +v_{t}^{2} s_{W}^{2}\left[-\frac{3 y_{Z}^{2}+2}{12 y_{Z}^{2}}-\frac{1}{12 y_{Z}^{4}} \ln y_{Z}^{2}+\left(\frac{7}{48}+\frac{1}{6 y_{Z}^{2}}+\frac{y_{Z}^{2}}{4}-\frac{3}{16\left(4 y_{Z}^{2}-1\right)}\right) \Psi\left(y_{Z}\right)\right],
\end{aligned}
$$

with $y_{Z}=m_{t} / M_{z}, a_{t}=1 /\left(2 s_{W} c_{W}\right), v_{t}=\left(1 / 2-4 s_{W}^{2} / 3\right) /\left(s_{W} c_{W}\right)$ and $c_{W}=\sqrt{1-s_{W}^{2}}=$ $M_{W} / M_{Z}$. The function $\Psi(x)$ can be found in Eq. (9). It is straightforward to obtain the expansion in the various kinematical regions. Thus we refrain from listing them explicitly. In Fig. 4(a) the exact result is plotted together with the expansions where in each region the same depth is chosen as in Eq. (10). The vertical dashed line marks the phenomenological result for $y_{Z}$ which is nicely approximated both from the $m_{t} \gg M_{Z}$ and the $m_{t} \approx M_{Z}$ region. However, it should be noted that both parameterizations for the $m_{t}=M_{h}$ expansion become instable below $1 / y_{Z} \approx 0.6$.

At two-loop order we consider the diagrams of Fig. 2] where the Higgs boson is replaced by the $Z$ boson. The reduction of the occuring integrals is in close analogy and in fact the same set of master integrals is necessary. Although the region $m_{t} \ll M_{Z}$ is phenomenologically not relevant we nevertheless present the results since it constitutes an important cross checks for the other kinematical limits. Furthermore, it is possible to obtain from this limit the result for the case of the bottom quark. We adopt the notation from Eq. (17) and denote the contribution from the $Z$ boson to $c_{v}^{\operatorname{mix}}$ by $c_{v}^{Z \text {,mix }}$. The additional subscript "0", "1a" or "1b" reminds on the kinematical region considered. 


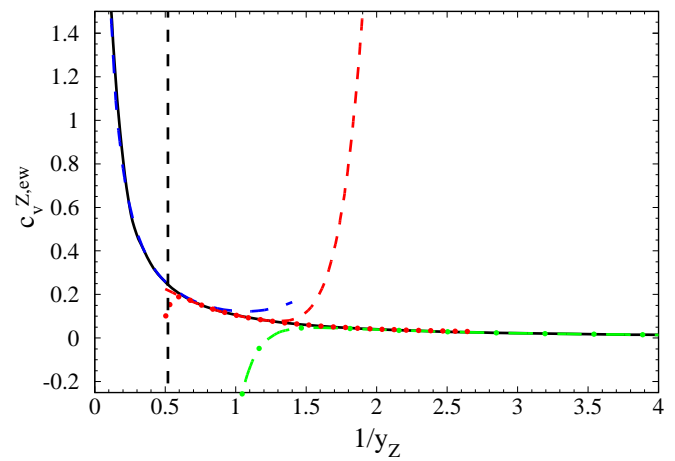

(a)

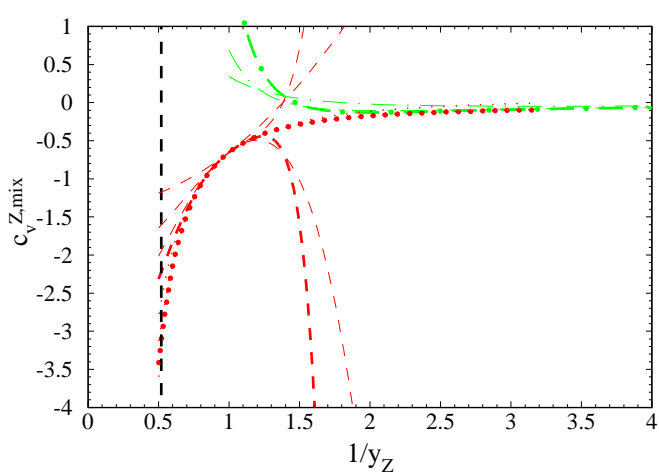

(b)

Figure 4: (a) $c_{v}^{Z, \text { ew }}$ and (b) $c_{v}^{Z \text {,mix }}$ as a function of $1 / y_{Z}$. The same notation as in Figs. 1 and 3 is adopted. The vertical line indicates the physical value of $1 / y_{Z}=M_{Z} / m_{t}$.

Our results read

$$
\begin{aligned}
c_{v, 0}^{Z, \text { mix }}= & \left(v_{t}^{2}+a_{t}^{2}\right) s_{W}^{2} \frac{\pi^{2}}{8} \ln \frac{m_{t}^{2}}{\mu^{2}} \\
& +v_{t}^{2} s_{W}^{2}\left[\left(-\frac{587}{432}+\frac{131 \pi^{2}}{576}-\frac{\pi^{2} \ln 2}{8}-\frac{21}{16} \zeta_{3}+\frac{28}{27} \ln y_{Z}^{2}-\frac{11}{72} \ln ^{2} y_{Z}^{2}\right) y_{Z}^{2}\right. \\
& +\left(-\frac{403}{64}+\frac{205 \pi^{2}}{576}+\frac{2299}{864} \ln y_{Z}^{2}-\frac{5}{18} \ln ^{2} y_{Z}^{2}\right) y_{Z}^{4} \\
& \left.+\left(-\frac{21091951}{864000}+\frac{3951 \pi^{2}}{2560}+\frac{28301}{7200} \ln y_{Z}^{2}+\frac{469}{160} \ln ^{2} y_{Z}^{2}\right) y_{Z}^{6}+\ldots\right] \\
& +a_{t}^{2} s_{W}^{2}\left[\left(-\frac{281}{432}+\frac{85 \pi^{2}}{192}-\frac{\pi^{2} \ln 2}{8}-\frac{21}{16} \zeta_{3}+\frac{29}{54} \ln _{Z}^{2}-\frac{11}{72} \ln ^{2} y_{Z}^{2}\right) y_{Z}^{2}\right. \\
& +\left(\frac{709}{576}+\frac{11 \pi^{2}}{432}+\frac{521}{288} \ln y_{Z}^{2}-\frac{79}{72} \ln ^{2} y_{Z}^{2}\right) y_{Z}^{4} \\
& \left.+\left(\frac{260687}{32000}-\frac{16013 \pi^{2}}{69120}+\frac{32453}{21600} \ln y_{Z}^{2}-\frac{5479}{1440} \ln ^{2} y_{Z}^{2}\right) y_{Z}^{6}+\ldots\right]
\end{aligned}
$$




$$
\begin{aligned}
c_{v, 1 a}^{Z, \text { mix }}= & \left(v_{t}^{2}+a_{t}^{2}\right) s_{W}^{2} \frac{\pi^{2}}{8} \ln \frac{m_{t}^{2}}{\mu^{2}} \frac{1}{1-y_{Z, 1 a}} \\
& +v_{t}^{2} s_{W}^{2}\left[-4.564-4.699 y_{Z, 1 a}-5.009 y_{Z, 1 a}^{2}-5.277 y_{Z, 1 a}^{3}-5.502 y_{Z, 1 a}^{4}\right. \\
& \left.-5.693 y_{Z, 1 a}^{5}+\ldots\right]+a_{t}^{2} s_{W}^{2}\left[-1.324-1.504 y_{Z, 1 a}-1.740 y_{Z, 1 a}^{2}\right. \\
& \left.-1.930 y_{Z, 1 a}^{3}-2.083 y_{Z, 1 a}^{4}-2.212 y_{Z, 1 a}^{5}+\ldots\right] \\
c_{v, 1 b}^{Z, \operatorname{mix}}= & \left(v_{t}^{2}+a_{t}^{2}\right) s_{W}^{2} \frac{\pi^{2}}{8} \ln \frac{m_{t}^{2}}{\mu^{2}}\left(1-y_{Z, 1 b}\right)+v_{t}^{2} s_{W}^{2}\left[-4.564+4.699 y_{Z, 1 b}\right. \\
& \left.-0.311 y_{Z, 1 b}^{2}-0.043 y_{Z, 1 b}^{3}+0.001 y_{Z, 1 b}^{4}+0.011 y_{Z, 1 b}^{5}+\ldots\right] \\
& +a_{t}^{2} s_{W}^{2}\left[-1.324+1.504 y_{Z, 1 b}-0.236 y_{Z, 1 b}^{2}-0.047 y_{Z, 1 b}^{3}-0.011 y_{Z, 1 b}^{4}\right. \\
& \left.-0.001 y_{Z, 1 b}^{5}+\ldots\right]
\end{aligned}
$$

where the divergence has been subtracted in a minimal way and $y_{Z}=m_{t} / M_{Z}, y_{Z, 1 a}=$ $\left(1-1 / y_{Z}^{2}\right)$ and $y_{Z, 1 b}=\left(1-y_{Z}^{2}\right)$. In Fig. 4(b) the results of Eq. (18) are shown as a function in $1 / y_{Z}$. Similarly to the two-loop case one observes a rapid convergence for $c_{v, 1 b}^{Z \text {,mix }}$ whereas the magnitude of the coefficients of the higher order terms in $c_{v, 1 a}^{Z \text {,mix }}$ even increase. This gives us quite some confidence that $c_{v, 1 b}^{Z, \text { mix }}$ evaluated at the physical scale provides a very good approximation to the unknown exact result. Using $1 / y_{Z}=91.19 / 175 \approx 0.52$ we get

$$
\left.c_{v}^{Z, \text { mix }}\right|_{\mu^{2}=m_{t}^{2}} \approx-3.1 \pm 0.3,
$$

where we assign a generous uncertainty of $10 \%$ which results from the comparison of lower-order approximations. The accuracy of the result for $c_{v}^{Z \text {,mix }}$ as given in Eq. (19) is more than sufficient as far as the forseen precision of the measurement of the threshold top quark production is concerned.

\section{B Addendum to Ref. [13]}

In this Appendix we want to provide an update of the results of Ref. [13]. In Fig. 4 of that reference the Higgs boson mass dependence of the on-shell wave function renormalization 


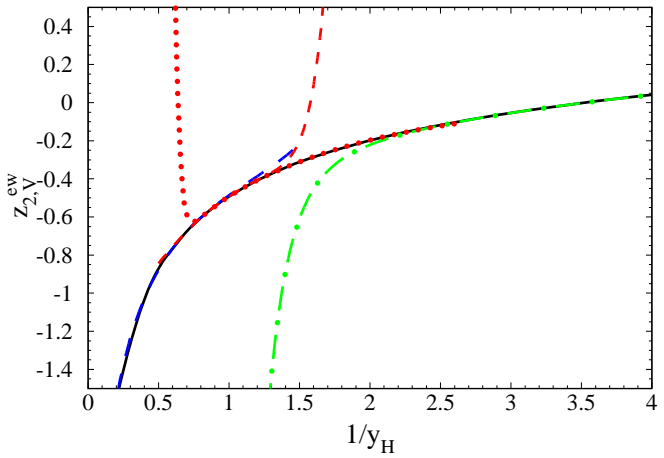

(a)

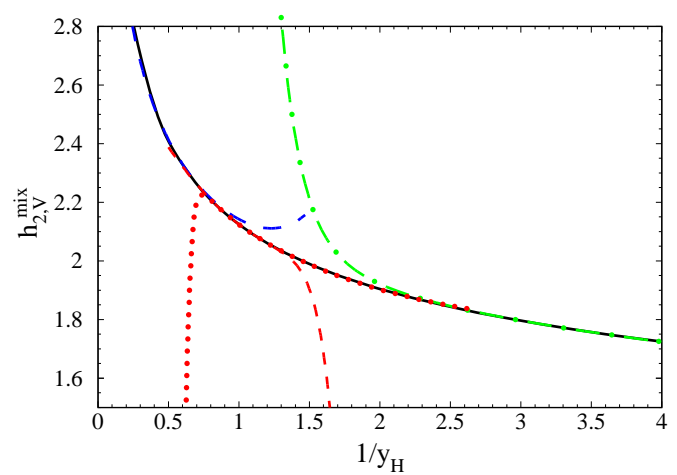

(b)

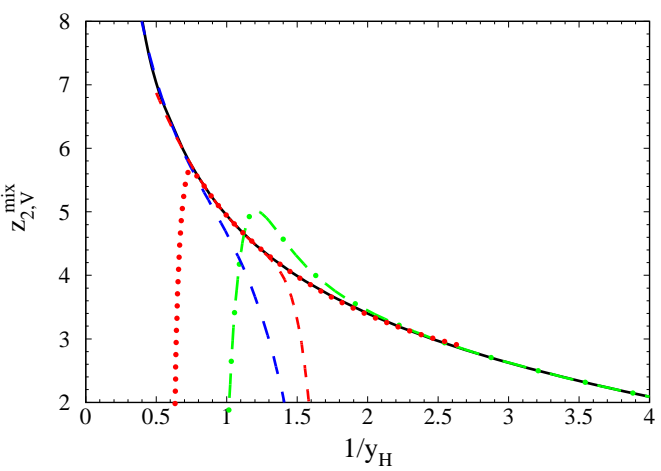

(c)

Figure 5: (a) One-loop, (b) 1/ $\epsilon$ pole and (c) constant part of the two-loop corrections to $Z_{2}^{V \text {,OS }}$ as a function of $1 / y_{H}=M_{H} / m_{t}$. The solid (black) line represents the exact result. The approximations in the three regions are shown as dotted, dashed and dash-dotted lines where for the expansion around $y_{H} \approx 1$ two different parameterizations (short dashes and dots) have been chosen.

constant to one- and two-loop order has been plotted as a function of $1 / y_{H}=M_{H} / m_{t}$. Next to the exact results also the ones for $m_{t} \ll M_{H}, m_{t} \approx M_{H}$ and $m_{t} \gg M_{H}$ have been shown and good agreement over almost the whole range in $y_{H}$ has been found. However, there was a small gap around $1 / y_{H} \approx 2$ which was not covered very well. This deficit is removed in Fig. [5 where the result obtained in the limit $m_{t} \approx M_{H}$ is plotted both in terms of $y_{H, 1 a}=\left(1-1 / y_{H}^{2}\right)$ (dashed) and $y_{H, 1 b}=\left(1-y_{H}^{2}\right)$ (dotted). One can see that the former is valid down to quite small values of $1 / y_{H}$ whereas the validity of the latter expansion reaches up to larger values of $1 / y_{H}$ leading to a significant overlap with the expansions for large and small values of $y_{H}$. Thus, in the whole $y_{H}$ range the simple expansions approximate the exact result to a very high precision.

Let us also mention that there is a misprint in the definition of $v_{t}$ in Ref. [13] after Eq. (17): it is too small by a factor two. Furthermore, the analytic expression for $Y^{\epsilon}$ in Eq. (22) has to be multiplied by $(-1)$ and in Eq. (27) a factor $1 / s_{W}^{2}$ is missing in the 
terms containing $\alpha_{s} / \pi$.

\section{References}

[1] see, e.g., http://linearcollider.org/

[2] M. Martinez and R. Miquel, Eur. Phys. J. C 27 (2003) 49.

[3] A. H. Hoang et al., Eur. Phys. J. directC 2 (2000) 1 arXiv:hep-ph/0001286.

[4] A. A. Penin, V. A. Smirnov and M. Steinhauser, Nucl. Phys. B 716 (2005) 303 arXiv:hep-ph/0501042.

[5] M. Beneke, Y. Kiyo and K. Schuller, Nucl. Phys. B 714 (2005) 67 arXiv:hep-ph/0501289.

[6] M. J. Strassler and M. E. Peskin, Phys. Rev. D 43 (1991) 1500.

[7] A. H. Hoang and C. J. Reisser, Phys. Rev. D 71 (2005) 074022 arXiv:hep-ph/0412258.

[8] A. H. Hoang and C. J. Reisser, arXiv:hep-ph/0604104.

[9] W. E. Caswell and G. P. Lepage, Phys. Lett. B 167 (1986) 437.

[10] G. T. Bodwin, E. Braaten and G. P. Lepage, Phys. Rev. D 51 (1995) 1125 [Erratumibid. D 55 (1997) 5853] arXiv:hep-ph/9407339.

[11] B. A. Kniehl, A. A. Penin, V. A. Smirnov and M. Steinhauser, Nucl. Phys. B 635 (2002) 357 arXiv:hep-ph/0203166.

[12] B. A. Kniehl, A. A. Penin, M. Steinhauser and V. A. Smirnov, Phys. Rev. Lett. 90 (2003) 212001 arXiv:hep-ph/0210161.

[13] D. Eiras and M. Steinhauser, JHEP 0602 (2006) 010 arXiv:hep-ph/0512099.

[14] M. Beneke and V. A. Smirnov, Nucl. Phys. B 522 (1998) 321 arXiv:hep-ph/9711391.

[15] V.A. Smirnov, Applied Asymptotic Expansions in Momenta and Masses, SpringerVerlag, Berlin-Heidelberg, 2001.

[16] R. J. Guth and J. H. Kühn, Nucl. Phys. B 368 (1992) 38.

[17] A. Czarnecki and K. Melnikov, Phys. Rev. Lett. 80 (1998) 2531 arXiv:hep-ph/9712222.

[18] M. Beneke, A. Signer and V. A. Smirnov, Phys. Rev. Lett. 80 (1998) 2535 arXiv:hep-ph/9712302. 
[19] B. A. Kniehl, A. Onishchenko, J. H. Piclum and M. Steinhauser, arXiv:hep-ph/0604072, Phys. Lett. B (in print).

[20] P. Nogueira, J. Comput. Phys. 105 (1993) 279.

[21] R. Harlander, T. Seidensticker and M. Steinhauser, Phys. Lett. B 426 (1998) 125 hep-ph/9712228.

[22] T. Seidensticker, hep-ph/9905298.

[23] C. Anastasiou and A. Lazopoulos, JHEP 0407 (2004) 046 arXiv:hep-ph/0404258.

[24] A. A. Penin and M. Steinhauser, Phys. Lett. B 538 (2002) 335 arXiv:hep-ph/0204290.

[25] F. Jegerlehner, arXiv:hep-ph/0105283. 